\title{
Temperature Gradient-Based DNA Computing for Graph Problems with Weighted Edges
}

\author{
Ji Youn Lee ${ }^{1}$, Soo-Yong Shin ${ }^{2}$, Sirk June Augh ${ }^{3}$, Tai Hyun Park ${ }^{1}$, and \\ Byoung-Tak Zhang ${ }^{2,3}$ \\ 1 Cell and Microbial Engineering Laboratory \\ School of Chemical Engineering, \\ 2 Biointelligence Laboratory \\ School of Computer Science and Engineering, \\ 3 Center for Bioinformation Technology (CBIT), \\ Seoul National University, Seoul 151-742, Korea \\ \{jylee, syshin, sjaugh, thpark, btzhang\}@bi.snu.ac.kr
}

\begin{abstract}
We propose an encoding method of numerical data in DNA using temperature gradient. We introduce melting temperature (Tm) for this purpose. Melting temperature is a unique characteristic to manipulate the hybridization and denaturation processes that used in the key steps in DNA computing such as the solution generation step and the amplification step. DNA strands of lower melting temperature tend to denature with ease and also be easily amplified by slightly modified polymerase chain reaction, called denaturation temperature gradient polymerase chain reaction. Using these properties, we implement a local search molecular algorithm using temperature gradient, which is contrasted to conventional exhaustive search molecular algorithms. The proposed methods are verified by solving an instance of the travelling salesman problem. We could effectively amplify the correct solution and the use of temperature gradient made the detection of solutions easier.
\end{abstract}

\section{Introduction}

Since Adleman's pioneering work 1], DNA computing has been applied to various fields of research including combinatorial optimization [9], massive parallel computing [7], Boolean circuit development [1], nanotechnology [17, huge database [12, and so on. Though many researches achieve the good results, most of these applications do not consider the problem of representing numerical data in DNA molecules. However, many real world applications involve graph problems which have weighted edges. Examples include the shortest path problem, the travelling salesman problem, the minimum spanning tree problem, and the Steiner tree problem [4. The numerical data representation method in DNA strands is the one of the important issue to extend the field of DNA computing. Though some researchers tried to represent the numerical data using DNA, the results are not satisfactory yet. 
In this paper, we propose a novel encoding method that uses temperature gradient to overcome the drawbacks of the previous works. We represent the numerical data using melting temperature gradient, and implement a local search molecular algorithm with the temperature gradient method. The travelling salesman problem (TSP) is used as a benchmark problem for the proposed technology. TSPs are interesting in that their solution requires representing the path weights. This is contrasted with the Hamiltonian path problem where the connection cost is binary. Our bio-lab experiments show that the correct DNA strands are effectively amplified and the detection of optimal solutions made easier.

In Section 2, we describe the molecular encoding of numerical data in DNA molecules. Section 3 explains the molecular algorithm and experimental procedure for solving TSP. Section 4 presents the results and discusses them. The conclusions are drawn in Section 5.

\section{Molecular Encoding for Edge Weights of Graph}

\subsection{Previous Encoding Methods}

Firstly, Narayanan and Zorbalas proposed DNA algorithms to solve travelling salesman problems [8]. Their method makes the length of weight sequences proportional to the edge costs in the edge sequences. This method is inefficient in representing a wide range of weights because, in some cases, one edge sequence with a high weight must be very long. For the same reason, it is also inefficient to represent real values. In addition, the fact that larger weights are encoded as longer sequences is contrary to the biological fact; the longer the sequences are, the more likely they hybridize with other DNA strands, though we have to find the shortest DNA strands.

Yamamura et al. proposed a concentration control method to represent the weights 18. The concentration of each DNA is used as input and output data, i.e. the numerical data is encoded by concentrations of DNAs. This method enables local search among all candidate solutions instead of exhaustive search. However, the concentration control method has some drawbacks in detecting the solutions. One cannot be sure that the most intensive band (in the gel) has the optimal solutions, because the most intensive band can be made by a number of partial non-optimal solutions with low concentration rather than optimal solutions with high concentration. In addition, it is technically difficult to extract a single optimal solution from the most intensive band.

In previous work [15], we proposed a method for representing real-values in fixed-length DNA strands using GC contents. Edge sequences contain two components: link sequences and weight sequences. The weight of edges is represented by varying the amount of $\mathrm{A} / \mathrm{T}$ pairs and $\mathrm{G} / \mathrm{C}$ pairs in weight sequences. Since there are two hydrogen bonds formed between $\mathrm{A}$ and $\mathrm{T}$ and three hydrogen bonds between $\mathrm{G}$ and $\mathrm{C}$, the hybridizations between the $\mathrm{G} / \mathrm{C}$ pairs are preferred to those between $\mathrm{A} / \mathrm{T}$ pairs. Therefore, the search procedure can be guided by including more A/T pairs to higher weight sequences and more $\mathrm{G} / \mathrm{C}$ 
pairs to smaller weight sequences. And we verified our method by the computer simulation.

\section{$2.2 \quad$ Melting Temperature Control Encoding}

Based on previous work, we improve the weight representation method by allowing the hybridization process to be controlled by melting temperature. Though the number of hydrogen bonds is an important factor deciding the thermal stability of DNA strands, it is not the only one. The melting temperature (Tm) is the more direct characteristic of the stability of the DNA hybrid and Tm is determined by more complex function consists of various factors including GC content. So, we adopt a melting temperature to encode the DNA strands.

Many methods have been proposed to determine the Tm value of DNA duplex. In the absence of destabilizing agents, like formamide or urea, Tm depends on three major parameters: the GC content and thermodynamic factors, the strand concentration, and the salt concentration. If we ignore the reaction conditions, Tm only depends on the GC content and thermodynamic factors. The classical method is using GC content, salt concentration, and the length [16]. But recently a statistical method using the thermodynamic parameters such as $\Delta H$ and $\Delta S$ is proposed [5] 10. The latter model is known as the nearest-neighbor $(\mathrm{NN})$ model that is more accurate and applicable to DNA duplex up to $108 \mathrm{bp}$.

We use both the GC content method and the nearest-neighbor method to calculate the Tm. If the sequences are short, the NN method is used, otherwise the classical method is mainly applied. Though Tm of the DNA duplex up to 108 bp can be accurately determined by the NN method, Tm of the longer DNA strands formed after hybridization and ligation depends on the classical method rather than the NN method.

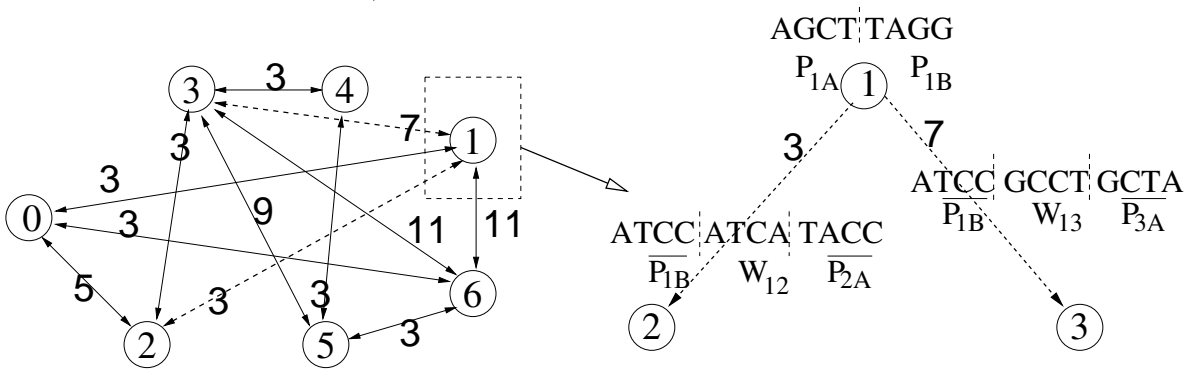

Fig. 1. Graph for the travelling salesman problem (left) and its example of encoding in DNA (right). The vertex sequence is $5^{\prime} \rightarrow 3^{\prime}$ and the edge sequence is $3^{\prime} \rightarrow 5^{\prime}$. The optimal path for this problem is ' $0 \rightarrow 1 \rightarrow 2 \rightarrow 3 \rightarrow 4 \rightarrow 5 \rightarrow 6 \rightarrow$ 0 .

The melting temperature control encoding scheme is illustrated for the travelling salesman problem in Fig. 1 Basic representation is similar to Adleman's 
method [1]. The difference is that edge sequences have the weight sequence part in the middle of their sequences, $W_{12}, W_{13}$ (Refer to the right part of Fig. 1). To design the sequences for weighted-graph problems using the proposed method, the vertex sequences are designed firstly. Each vertex sequence is designed to have a similar Tm using both the nearest-neighbor and the classical method. That is why the vertex sequences should not affect the hybridization fidelity. Then, the edge sequences are generated. The edge sequence consists of the two parts such as the link sequence $\left(\overline{P_{1 B}}, \overline{P_{2 A}}, \overline{P_{3 A}}\right)$ and the weight sequence $\left(W_{12}\right.$, $\left.W_{13}\right)$. This scheme is motivated from [9]. The link sequences are based on the vertex sequences to be linked and have also a similar Tm, since the link sequences of the edge sequences are decided by the vertex sequence. The weight sequences are designed to have a various Tm according to their representative cost. To produce more variation in generating low cost paths, the weight sequences with smaller weights have a lower Tm. If DNA strands have a lower Tm, it can be easily denatured and also easily amplified by denaturation temperature gradient polymerase chain reaction. For example, as shown in Fig. 1, the first part $\left(\overline{P_{1 B}}\right)$ of the edge sequence is complementary to the last half $\left(P_{1 B}\right)$ of the starting vertex sequence. And the last part $\left(\overline{P_{2 A}}\right)$ is complementary to the first half of the ending vertex sequence $\left(P_{2 A}\right)$. The weight sequence $W_{12}$ with cost 3 contains more $\mathrm{A} / \mathrm{T}$ pairs than the weight sequence $W_{13}$ with cost 7 to lower the Tm.

\subsection{Travelling Salesman Problems}

We selected travelling salesman problem as a benchmark. The travelling salesman problem (TSP) is to find a minimum weight (cost) path for a given set of vertices (cities) and edges (roads). In addition, the solution path must contain all the cities given, each only once, and begin from the specified city to which the tour ends [4]. We solve the TSP with 7 nodes, 23 edges and 5 weights as shown in Fig. 1. For convenience, we represent the weights by decimal numbers such as $3,5,7,9$, and 11 . The example of DNA sequences for solving TSP is shown in Table 1. These sequences are generated by the NACST sequence generator (NACST/Seq) [6].

The sequences are generated to prevent mis-hybridization and unexpected secondary structure using seven constraints such as similarity, H-measure, Hmeasure in the 3'-end, GC ratio, continuity, the unexpected secondary structure, and melting temperature [14, 13]. For the vertex sequences, Tm ranges $55^{\circ} \mathrm{C}$ to $60^{\circ} \mathrm{C}$ using the NN model with $1 M$ salt concentration and $10 n M$ oligomer concentration, GC content is restricted to $50 \%$, the unexpected secondary structure such as hairpin formation is prohibited. In the weight sequences, Tm is increased by the cost; GC content is varied from $30 \%$ to $70 \%$ (see Table11). To avoid the extreme case, i.e. the sequence consists of all $\mathrm{C}$ or $\mathrm{G}$, we set the range as $30 \%$ $\sim 70 \%$. As the result of evolutionary algorithms, NACST/Seq assigns the $30 \%$ to weight $3,40 \%$ to weight 5 , and so on. NACST/Seq increases GC content by $10 \%$, as the weight is increased by two. After hybridization and ligation, the long sequences are generated which are not fit to the NN model. Therefore, evolutionary algorithm changed the GC ratio more precisely than melting temperature. 
Table 1. Vertex sequences and weight sequences for TSP. Tm is calculated by the NN method with $1 M$ salt concentration and $10 n M$ oligomer concentration.

\begin{tabular}{c|c|c|c}
\hline \multicolumn{4}{c}{ Vertex sequences } \\
\hline No. & Sequence $\left(5^{\prime} \rightarrow 3^{\prime}\right)$ & Tm & GC\% \\
\hline 0 & AGGCGAGTATGGGGTATATC & 60.73 & 50 \\
1 & CCTGTCAACATTGACGCTCA & 59.24 & 50 \\
2 & TTATGATTCCACTGGCGCTC & 59.00 & 50 \\
3 & ATCGTACTCATGGTCCCTAC & 56.81 & 50 \\
4 & CGCTCCATCCTTGATCGTTT & 58.13 & 50 \\
5 & CTTCGCTGCTGATAACCTCA & 59.44 & 50 \\
6 & GAGTTAGATGTCACGTCACG & 56.97 & 50 \\
\hline \multicolumn{4}{|c}{ Weight sequences } \\
\hline Edge cost & Sequence $\left(5^{\prime} \rightarrow 3^{\prime}\right)$ & Tm & GC\% \\
\hline 3 & ATGATAGATATGTAGATTCC & 47.89 & 30 \\
5 & GGATGTGATATCGTTCTTGT & 54.62 & 40 \\
7 & GGATTAGCAGTGCCTCAGTT & 58.37 & 50 \\
9 & TGGCCACGAAGCCTTCCGTT & 64.51 & 60 \\
11 & GAGCTGGCTCCTCATCGCGC & 68.88 & 70 \\
\hline
\end{tabular}

Additionally, the higher weight sequences have higher melting temperatures with the NN method.

\section{Molecular Algorithms for TSP}

\subsection{Previous Work}

Most of the existing DNA computing methods to solve combinatorial optimization problems are similar to Adleman's in several ways. First, all possible solutions are generated and then the correct solutions among them are selected. Also the previous works [8, 15, 18, on travelling salesman problems or shortest path problems are similar to each other. They only differ in their weight encoding schemes such as sequence length, GC content, and strand concentration. The former two studies were not verified by lab experiments, the last method is proved by lab experiments, though the results are not satisfactory. And last method [18] gives a bias to search space by concentration control.

\subsection{Temperature Gradient Algorithms}

In order to utilize the massive parallelism of biochemical materials and to guide search space, it is useful to give some biases in the experimental steps. For this purpose, we give some constraints in DNA concentration and the PCR step. By varying the concentration of each oligomer strands [18, we can generate more paths that contains a smaller sum of weights.

Also by modifying the PCR protocol, we can specifically amplify and detect the correct solution easier. DNA strands of low melting temperature can 
be more strongly amplified in PCR by varying the denaturation protocol. Usually, we vary the annealing temperature to optimize the reaction condition. But by denaturing the dsDNA in the lower temperature in the starting cycles and gradually increasing the denaturation temperature, we can specifically amplify the DNA strands with a lower melting temperature. So we can amplify the correct solutions more than other solutions that have the same length but a higher melting temperature.

The whole procedure to solve TSP is shown in Fig. 2 In step 1, we generate the solution set. Steps $2 \sim 5$ find the solution satisfying the restrictions (the starting vertex is the same as the end vertex). Step 6 separates the sequences visiting all vertexes. In steps $7 \sim 8$, we amplify the solutions based on its path cost and find the optimal solution. Finally, we check the path of the solution.

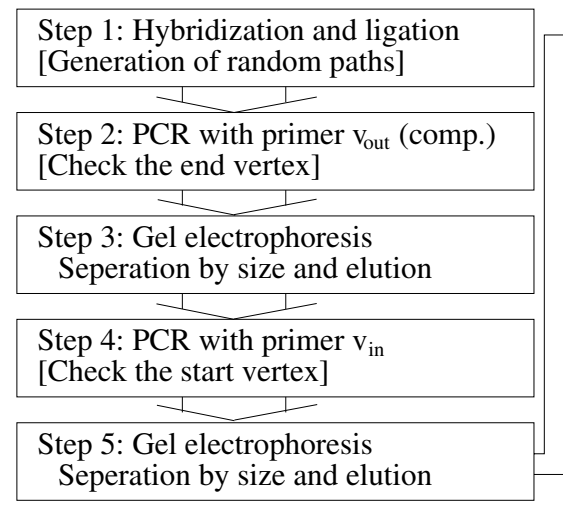

Step 6: Affinity-separation with
a biotin-avidin magnetic beads system
[Check the each vertex]

Step 7: DTG-PCR with $\mathrm{v}_{\text {in }}$ and $\mathrm{v}_{\text {out }}$ (comp.)

[Amplify the correct solutions more]

Step 8: Gel electrophoresis separation
by size and Tm: TGGE
1

Step 9: Sequencing and readout

Fig. 2. The molecular algorithms for solving TSP.

Oligomer Synthesis All 7 vertexes and 5 weights are designed in 20 mer ssDNA as listed in Table 1. And 23 edges in 40 mer ssDNAs are designed according to their vertexes and weight sequences. All oligomers are 5'-phosphorylated. Other 5'-biotinylated vertexes were prepared for affinity-separation. All oligomers were synthesized at Bioneer Co.

Hybridization and Ligation Edge and weight oligomers were differently added by their weights. The amount decreased by $20 \%$ according to the weight increase by 2 , set on the basis of weight 3 as a $100 \%$. Oligomer mixture was heated to $95^{\circ} \mathrm{C}$ and slowly cooled to $20^{\circ} \mathrm{C}$ by $1^{\circ} \mathrm{C}$ per 1 minute, and stored at $4^{\circ} \mathrm{C}$.

Mix $5 \mu l$ of hybridization mixtures and 350 units of T4 DNA ligase (TaKaRa, Japan), ligase buffer (66m $M$ Tris-HCl, pH 7.6, 6.6m $\mathrm{MgCl}_{2}, 10 \mathrm{mM}$ DTT, $0.1 \mathrm{mM}$ ATP), and add $\mathrm{H}_{2} \mathrm{O}$ to a total volume of $10 \mu \mathrm{l}$. We incubated the reaction mixture for 16 hours at $16^{\circ} \mathrm{C}$. 
PCR Amplification and Gel Electrophoresis All PCR amplifications were performed on a PTC-200 DNA Engine (MJ Research, MA, USA). For normal amplification, $0.5 \mu M$ of primer and AccuPower PCR PreMix (Bioneer, Korea) which containing 1 unit of Taq DNA polymerase in $10 \mathrm{mM}$ Tris- $\mathrm{HCl}$, pH 9.0, $1.5 \mathrm{mM} \mathrm{MgCl} \mathrm{M}_{2}, 40 \mathrm{mM} \mathrm{KCl}, 0.25 \mathrm{mM}$ each dNTP were dissolved to distilled water to a total volume of $20 \mu \mathrm{l}$. And PCR was processed for 34 cycles at $95^{\circ} \mathrm{C}$ for 30 seconds, at $(T m-5)^{\circ} \mathrm{C}$ for 30 seconds and at $72^{\circ} \mathrm{C}$ for 30 seconds. Initial denaturation and prolonged polymerization was executed for 5 minutes.

All gel electrophoresis were performed with 2\% Agarose-1000 (GibcoBRL, NY, USA) in 0.5X tris-borate-EDTA buffer and the gel was ethidium bromide stained. 50bp DNA Ladder (GibcoBRL, NY, USA) was used as a marker.

Affinity Separation We roughly followed the affinity separation protocol in the Adleman's experiments. The ssDNA for the affinity purification were produced by replacing the forward primer with 5'-biotinylated analog. The amplified product was annealed to streptavidin paramagnetic particles (Promega, Madison, WI) by incubating in $200 \mathrm{ml}$ of $0.5 \times$ saline sodium citrate (SSC) for 1 hour at room temperature with constant shaking. Particles were washed three times in $300 \mathrm{ml}$ of $0.5 \times \mathrm{SSC}$ and then heated to $80^{\circ} \mathrm{C}$ in $100 \mathrm{ml}$ of $d d \mathrm{H}_{2} \mathrm{O}$ for 5 minutes to denature the bound dsDNA. The aqueous phase with ssDNA was retained. For affinity separation, $1 \mathrm{nmol}$ of 5'-biotinylated vertex strands was annealed to particles as above and washed three times in $300 \mathrm{ml}$ of $0.5 \times \mathrm{SSC}$ for 1 hour at room temperature with constant shaking. Particles were washed four times in $300 \mathrm{ml}$ of $0.5 \times \mathrm{SSC}$ to remove unbound ssDNA and then heated to $80^{\circ} \mathrm{C}$ in $100 \mathrm{ml}$ of $d \mathrm{dH}_{2} \mathrm{O}$ for 5 minutes to release ssDNA bound to the complementary vertex 1 . The aqueous phase with ssDNA was retained. This process was then repeated with each vertexes.

Denaturation Temperature Gradient PCR For denaturation temperature gradient PCR (DTG-PCR), the denaturation temperature was kept initially $70^{\circ} \mathrm{C}$ and gradually decreased $1^{\circ} \mathrm{C}$ per one cycle and kept $95^{\circ} \mathrm{C}$ for the remaining 10 cycles. Other conditions are identical with normal PCR described in the PCR amplification section.

Temperature Gradient Gel Electrophoresis For TGGE analysis, we used $8 \%$ denaturing polyacrylamide gel containing $20 \%$ formamide and $4.2 \mathrm{M}$ urea. The electrophoresis was performed over $55^{\circ} \mathrm{C} \sim 70^{\circ} \mathrm{C}$ temperature range at 200 volt for three hours. After the electrophoresis, the gel was silver stained.

Cloning and Sequencing After the TGGE, the DNA strands in main band were excised, eluted and PCR amplified. This PCR product was cloned into pBluescript SK+ and sequenced using $\mathrm{T} 7$ primer. 


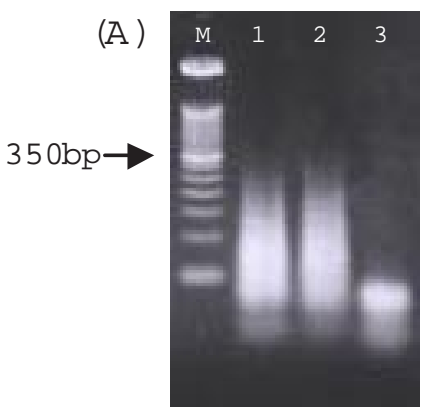

(B)

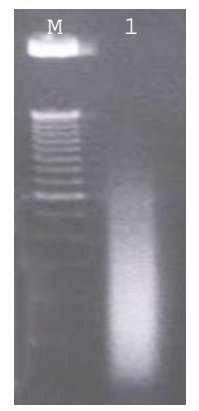

(C)

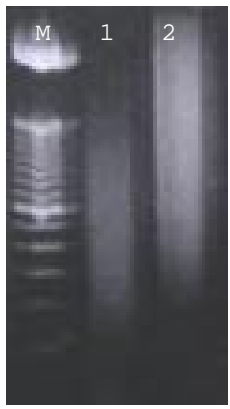

(D)
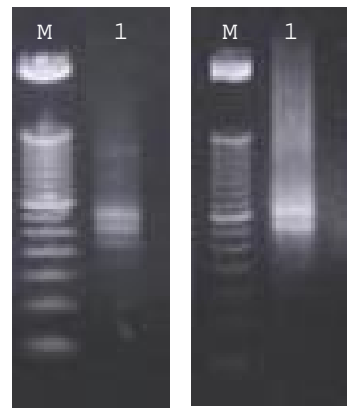

Fig. 3. Gel electrophoresis on a $2 \%$ agarose gel. The lanes contain: lane M denotes DNA size marker (50 bp ladder), (A) lanes 1,2: first ligation result, lane 3: oligomer mixture, (B) lane 1: second ligation result, (C) lanes 1,2: first PCR with $V_{\text {out }}$ result, (D) lane 1: second PCR with $V_{\text {in }}$ result

\section{Results and Discussion}

\subsection{Random Path Formation and Size Sieving}

The start point to solve TSP is generating initial pool, random path formation. Searching a correct solution in the candidate pool is an important step to solve the problem. However, more generation of the possible solution in the candidate pool is readily achievable if we utilize the advantages of molecular computing. The reaction rate of biochemical reaction is related with the reaction constant and the reactant concentrations. Therefore, concentration gradient can be useful to obtain the correct solution. This approach was already tried by [18. By increasing the concentration of DNA sequences of smaller weights and vice versa, paths which contains a smaller sum of weights will be more frequently generated. In our work, the concentration gradient was only a tool to generate more possible solutions.

The hybridization and ligation results are shown in Fig. 3 (A). Compared with the oligomer mixture, the ligated DNA strands became elongated. But, 
there are few copies around the $300 \mathrm{bp}$ that indicates the length of paths including 8 vertexes. So we executed the ligation reaction again and obtained an upper shifted ligation product as shown in Fig. 3 (B). However, still shorter DNA strands mainly occupy the ligation product. Ligation is an essential step to generate random paths, so efficient ligation is needed to produce DNA strands long enough to solve large problems.

\subsection{PCR with In and Out Vertex and Affinity Purification}

After the hybridization and ligation, double-stranded DNAs with sticky ends were generated. We cannot use the primer pair because the start and the end point are identical in TSP and the primer pair is exactly complementary to each other. So we executed PCRs with only one primer. First, we executed PCR with a primer that is complementary with the end vertex (vertex 0). Blunt-ended double-stranded DNAs that end with the vertex 0 will be generated by this PCR. Subsequently, we executed the second PCR with vertex 0 , and we could amplify the DNAs that start with vertex 0 . We sieved the PCR product by $2 \%$ agarose gel electrophoresis (Fig. 3(C), (D)). The second PCR product appeared in several bands in the gel. We excised and eluted the band around $300 \mathrm{bp}$ and amplified with 5'-biotinylated vertex 0 as a primer. The amplified product was used in the following affinity separation with streptavidin paramagnetic particles.

\subsection{Denaturation Temperature Gradient PCR}

The DNA strands that passed the implementation procedures up to step 6 have the same length and contains same oligomer segments. So, they have similar characteristics and cannot be easily separated. However, we designed the weight sequence to have different GC content, so the Tm of the correct solution is the lowest among the candidate solutions. Therefore, if we decrease the denaturation temperature to a certain level, mainly DNA strands of correct solutions will be denatured at that temperature, and be amplified. As the denaturation temperature increases, other DNA strands also will be amplified. But the amount of correct solutions will be exponentially increased cycle by cycle, and occupy the major part of the solution.

By simple modification of the typical PCR, we can amplify the correct solution and detect it easily. We can show the effectiveness of DTG-PCR by the relative amplification in Fig. 4(A). Shorter DNA strands in the lane 2 are more amplified by DTG-PCR when compared with the normal PCR product in the lane 1 (See the boxed area in Fig. 4). After the DTG-PCR, we obtained the DNA strands as shown in amplification in Fig. 4(B). This band might contains four different DNA strands of the possible Hamiltonian paths, ' $0 \rightarrow 1 \rightarrow 2 \rightarrow 3 \rightarrow 4 \rightarrow$ $5 \rightarrow 6 \rightarrow 0$ (the sum of weights: 21 )', ' $0 \rightarrow 1 \rightarrow 6 \rightarrow 5 \rightarrow 4 \rightarrow 3 \rightarrow 2 \rightarrow 0$ (31)', ' $0 \rightarrow 2 \rightarrow 1 \rightarrow 3 \rightarrow 4 \rightarrow 5 \rightarrow 6 \rightarrow 0(27)^{\prime}$ ', $0 \rightarrow 2 \rightarrow 3 \rightarrow 4 \rightarrow 5 \rightarrow 6 \rightarrow 1 \rightarrow 0$ (31)'. Those strands have the identical length, and they cannot be separated by normal gel electrophoresis. However, we can separate these strands by the temperature gradient gel electrophoresis (TGGE), because they have distinct 
(A)

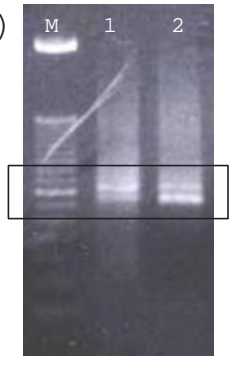

(B)

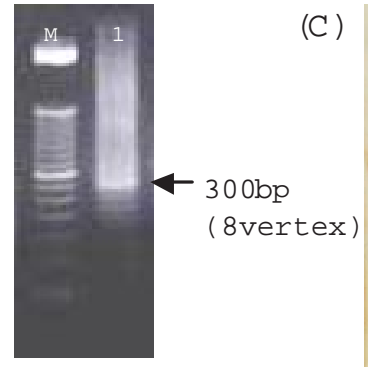

(C)

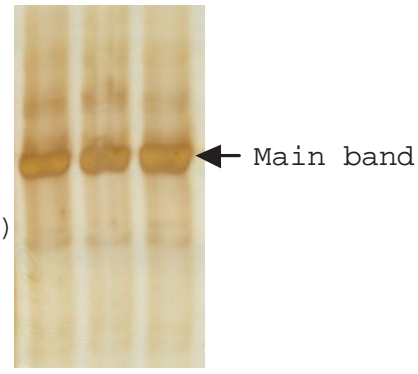

Fig. 4. Gel electrophoresis on a $2 \%$ agarose gel. The lanes contain: lane M denotes DNA size marker (50 bp ladder), (A) lane 1: normal PCR result, lane 2: DTG-PCR result, (B) lane 1: the final DTG-PCR result, (C) TGGE separation of solutions

melting behaviors. Similar work has studied using denaturing gradient gel electrophoresis (DGGE) [3].

\subsection{Temperature Gradient Gel Electrophoresis and Sequencing}

TGGE is analogous to DGGE and it is based on the correlation of the melting characteristic of a DNA strand. TGGE is an extremely sensitive method and even fragments differing in only one nucleotide in sequence can be separated by TGGE. Usually this property was used to detect point mutations. But we utilized this electrophoresis method to separate the correct solution from other possible Hamiltonian paths. The GC content of the strands varies from $40.67 \%$ to $44.00 \%$, and the melting temperature of the DNA strands are respectively $84.80^{\circ} \mathrm{C}, 86.51^{\circ} \mathrm{C}, 85.68^{\circ} \mathrm{C}, 86.62^{\circ} \mathrm{C}$ by $\mathrm{NN}$ model [5] with $1 M$ salt concentration and $10 n M$ oligomer concentration, and $92.69^{\circ} \mathrm{C}, 94.05^{\circ} \mathrm{C}, 93.51^{\circ} \mathrm{C}, 94.05^{\circ} \mathrm{C}$ by GC content method. The PCR mixture containing those possible Hamiltonian paths was separated by TGGE, and one main band and other sub bands were observed in Fig 4 (C). The DNA strands in main band were cloned and sequenced, and it showed that the path $0 \rightarrow 1 \rightarrow 2 \rightarrow 3 \rightarrow 4 \rightarrow 5 \rightarrow 6 \rightarrow 0$ has the lowest cost. The sequencing result confirmed the whole experimental procedures.

\subsection{Scalability}

The solvable problem size is restricted to the capabilities of the current biochemical tools. In case of solving TSP, PCR is the critical step in the procedure. We can amplify DNA strand of up to 40,000 bases with current PCR technology. Though considering this limitation, we can scale up to 1,000 cities problems roughly, if we apply this temperature gradient encoding method. And The another important point of scalability is the ability of separation and detection. We 
can separate DNA strands whose GC content differs by $0.49 \%$ with the current resolution of TGGE and this difference is easily achievable with our encoding method.

However, there exist some limitations in scaling up to 1,000 city problems. The first and critical problem is that it is impossible to discriminate the solutions which have the identical melting temperatures. And it is not also discriminative when there exist two solutions that melting temperature differences are not exceeding $0.2^{\circ} \mathrm{C}$. The second problem is the presence of elaborate and timeconsuming implementation steps. In the experimental view, 1,000 times affinity separation is almost impossible. This is the most time-consuming step in our implementation steps. But, if we introduce the affinity separation method proposed by [2], the affinity separation steps can be automated with high efficiency and can became easier. Other possible obstacle in our encoding method arises when the DNA strands with stable mismatches have lower melting temperature and are amplified exclusively. So the sophisticated sequence design to avoid the stable mismatches is needed.

\section{Conclusions}

We introduced a temperature gradient method to solve the graph problems with numerical data. This method can overcome the restrictions of other encoding methods and be easily implemented by the simple modification of the existing experimental techniques. We can more strongly amplify the correct solution relatively during the reaction and the solution can be easily detected. This method drives the DNA pool to contain more correct solutions, rather than to search randomly for the correct solution in the DNA pool. We showed the feasibility of our computing method by solving the travelling salesman problem with adequate biochemical tools.

The combination of the orthogonal design of DNA sequences and denaturation temperature gradient PCR provides a novel method to solve general graph problems with weighted edges. This is applicable to any Tm-involved implementation steps to amplify low melting temperature DNA strands.

\section{Acknowledgments}

This research was supported in part by the Ministry of Education \& Human Resources Development under the BK21-IT Program and the Ministry of Commerce, Industry and Energy through the Molecular Evolutionary Computing (MEC) Project. The RIACT at Seoul National University provides research facilities for this study.

\section{References}

[1] L. M. Adleman. Molecular computation of solutions to combinatorial problems. Science, 266:1021-1024, 1994. 
[2] R. S. Braich, N. Chelyapov, C. Johnson, P. W. K. Rothemund, and L. Adleman. Solution of a 20-varaible 3-SAT problem on a DNA computer. Science, 296:499$502,2002$.

[3] J. Chen, E. Antipov, B. Lemieux, W. Cedeño, and D. H. Wood. In vitro selection for a MAX 1s DNA genetic algorithm. In Proceedings 5th International Workshop on DNA-Based Computers, pages 23-46, 2000.

[4] M. R. Garey and D. S. Johnson. Computers and Intractability: A Guide to the Theory of NP-completeness. W. H. Freeman and company, 1979.

[5] J. SantaLucia Jr. A unified view of polymer, dumbbell, and oligonucleotide DNA nearest-neighbor thermodynamics. Proc. Natl. Acad. Sci. USA, 95:1460-1465, 1998.

[6] D. Kim, S.-Y. Shin, I.-H. Lee, and B.-T. Zhang. NACST/Seq: A sequence design system with multiobjective optimization. In Preliminary Proceedings of 8th International Meeting on DNA Based Computer, pages 241-251, 2002.

[7] Q. Liu, L. Wang, A. G. Frutos, A. E. Condon, R. M. Corn, and L. M. Smith. DNA compouting on surface. Nature, 403:175-179, 2000.

[8] A. Narayanan and S. Zorbalas. DNA algorithms for computing shortest paths. In Proceedings of Genetic Programming 1998, pages 718-723, 1998.

[9] Q. Ouyang, P. D. Kaplan, S. Liu, and A. Libchaber. DNA solution of the maximal clique problem. Science, 278:446-449, 1997.

[10] R. Owczarzy, P. M. Vallone, F. J. Gallo, T. M. Paner, M. J. Lane, and A. S. Benight. Predicting sequence-dependent melting stability of short duplex DNA oligomers. Bioploymers, 44:217-239, 1998.

[11] G. G. Owenson, M. Amos, D. A. Hodgson, and A. Gibbsons. DNA-based logic. Soft Computing, 5(2):102-105, 2001.

[12] J. H. Reif, T. H. LaBean, M. Pirrug, V. S. Rana, B. Guo, C. Kingsford, and G. S. Wickham. Experimental construction of a very large scale DNA database with associatice search capability. In The 7th International Workshop on DNA-Based Computers, pages 241-250.

[13] S.-Y. Shin, D. Kim, I.-H. lee, and B.-T. Zhang. Multiobjective evolutionary algorithms to design error-preventing DNA sequences. Technical Report BI-02-003, Seoul National University, School of Computer Science and Engineering, Seoul National University, Seoul, Korea, March 2002.

[14] S.-Y. Shin, D.-M. Kim, I.-H. Lee, and B.-T. Zhang. Evolutionary sequence generation for reliable DNA computing. In Proceedings of Congress on Evolutionary Computation 2002, pages 79-84, 2002.

[15] S.-Y. Shin, B.-T. Zhang, and S.-S. Jun. Solving traveling salesman problems using molecular programming. In Proceedings of Congerss on Evolutionary Computation 1999, pages 994-1000, 1999.

[16] J. G. Wetmur. DNA probes: applications of the principles of nucleic acid hybridization. Crit. Rev. Biochem. Mol. Biol., 26:227-259, 1991.

[17] E. Winfree, F. Lin, L. A. Wenzler, and N. C. Seeman. Design and self-assembly of two-dimensional DNA crystals. Nature, 394(6693):539-545, 1998.

[18] M. Yamamura, Y. Hiroto, and T. Matoba. Solutions of shortest path problems by concentration control. In Proceedings of 7th International Workshop on DNABased Computers, pages 231-240, 2001. 